\section{Excitation of the 2s State of Atomic Hydrogen by Electron Impact}

IN a recent publication Kingston, Moiseiwitsch and Skinner have applied the second Born approximation, retaining only terms up to the third order in the interaction energy, to the calculation of the total cross-section $Q(1 s-2 s)$ for the $1 s-2 s$ excitation of hydrogen atoms by electrons. Their results are displayed in Fig. 1. Curve 1 is the cross-section obtained by using the first Born approximation while curves 4 and 6 are respectively the cross-sections given by the second Born approximation with allowance for: (a) distortion due to the coupling to the $1 s$ and $2 s$ states; $(b)$ polarization due to the coupling to the $2 p$ states as well as distortion due to the $1 s$ and $2 s$ states.

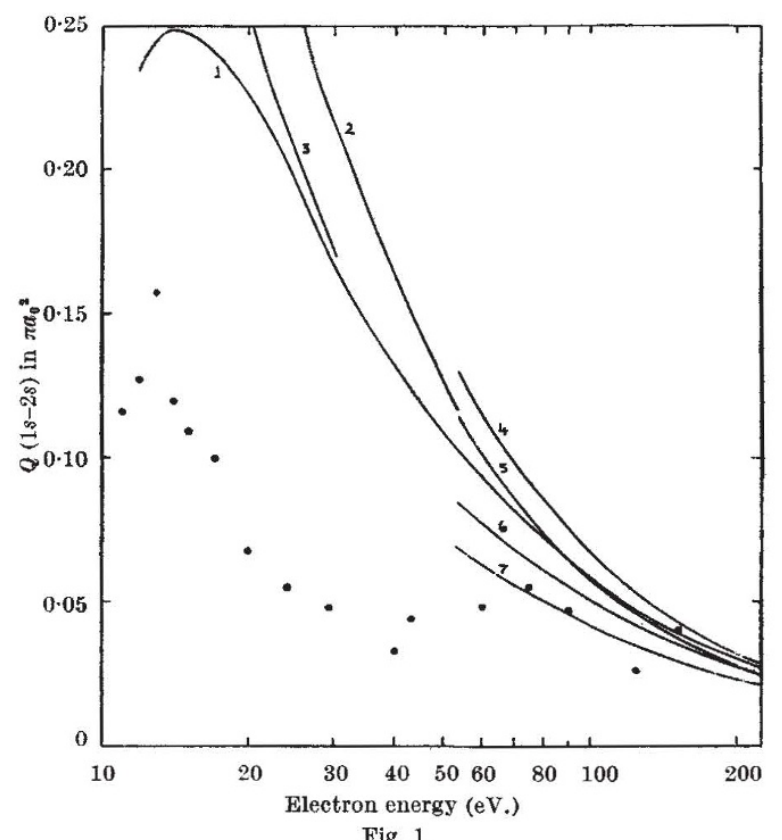

Fig. 1

$\mathrm{Wu}^{2}$ has reported first Born approximation calculations of $Q(1 s-2 s)$ in which allowance for exchange has been made. From the tables given by $\mathrm{Wu}$ the contribution resulting from exchange can be readily calculated and incorporated into the second Born approximation calculations of Kingston, Moiseiwitsch and Skinner. The resulting cross-sections are displayed in Fig. 1. Curve 5 includes the effects of distortion and exchange while curve 7 includes in addition the effect of polarization. Allowance for exchange reduces the excitation cross-section. Smith, Miller and Mumford ${ }^{3}$ have carried out exact solutions of the coupled differential equations for the $1 s-2 s$ dectron impact excitation of atomic hydrogen derived by making full allowance for the distortion due to the $1 s$ and $2 s$ states but neglecting exchange. The cross-section obtained by them is given by curve 2 and is in satisfactory accord with the second Born approximation curve 4 calculated by Kingston, Moiseiwitsch and Skinner.

Smith $^{4}$ has solved the coupled integro-differential equations for the $s, p, d$ partial waves making full allowance for exchange as well as distortion. The resulting total cross-section $Q(1 s-2 s)$ is given by curve 3, the higher-order partial cross-sections having been estimated with the first Born approximation. Curve 3 lies somewhat below curve 2, and thus allowance for exchange reduces the cross-section in agreement with the previously noted effect of exchange on the second Born approximation crosssections.

The experimental points of Stebbings, Fite, Hummer and Brackmann ${ }^{5}$ for $Q(1 s-2 s)$ are also dis played in the figure. Lichten ${ }^{6}$ has shown that the original data of Stebbings et al. are in error and should be multiplied by a factor $3 / 2$. This correction was therefore introduced (ef. Hummer and Seaton ${ }^{7}$ ).

There is satisfactory agreement, at least at energies greater than $60 \mathrm{eV}$., between the modified experimental results of Stebbings et al. and the cross-section curve 7 in which allowance for exchange as well as distortion and polarization has been included. Noting that curve 4 lies slightly above curve 2 , it is to be anticipated that curve 7 is slightly in excess of the actual $1 s-2 s$ excitation cross-section, which would then bring calculation into still closer agreement with the absolute experimental data of Stebbings et al.

The experimental cross-section obtained by Lichten and Schultz ${ }^{8}$ does not extend above 45 eV., and in any event is not absolute. Hence no comparison between theory and their experimental results is feasible. However, their relative cross-section is in good agreement with the shape of the cross-section obtained by Stebbings et al. as was pointed out by the latter authors.

\section{B. L. Moiseiwitsch}

Department of Applied Mathematies,

The Queen's University of Belfast.

${ }^{1}$ Kingston, A. E., Moiseiwitsch, B. L., and Skinmer, B. (.., Proc. Roy. Soc., A, $258,245(1960)$

${ }^{2}$ Wu, T. X., Canad. J. Phys., 38, 1654 (1960).

${ }^{3}$ Smith, K., Miller, W. F., and Mumford, A. J. P., Proc. Phys, Soc. 76, 559 (1960).

${ }^{4}$ Smith, K., Phys. Rev., 120, 845 (1960).

$\checkmark$ Stebbings, R. F., Fite, W. L., Hummer, D. G., and Brackmann. R. T., Phys. Rev., 119, 1939 (1960).

' Lichten, W., Phys. Rev. Letters, 6, 12 (1961).

'Hummer, D. G., and Seaton, M. J., Phys. Rev. Letters, 6, 471 (1961).

${ }^{8}$ Lichten, W., and Schultz, S., Phys. Rev., 116, 1132 (1959).

\section{Measurement of Stopping-Power Ratio for Cavity lonization Chambers}

The energy absorbed in a medium per unit mass, $E_{m}$, exposed to X- or $\gamma$-radiation can be related to the ionization produced per unit mass of gas, $J_{m}$, situated, in a cavity in the medium by the Bragg-Gray equation:

$$
E_{m}=\frac{1}{F_{m}} \cdot W J_{m}
$$

where $W$ is the average energy associated with the production of one ion pair in the gas, $F_{m}$ is the mass stopping-power ratio of the gas to the solid for electrons. $\quad F_{m}$ has been evaluated theoretically by assuming electrons lose energy continuously ${ }^{1,2}$ and also by allowing for discrete losses of energy ${ }^{3,4}$. However, experimental investigation has shown that these theoretical treatments are only useful for a limited range of size of cavity ${ }^{5}$, as indeed the initial assumptions in their derivations imply. In many situations the greatest uncertainty in measuring exposure or absorbed dose arises from uncertainty in this factor. Thus a direct measurement of $F_{m}$ is desirable.

Several workers have measured the relative stopping-power ratio, but Whyte ${ }^{6}$ devised a method 